\title{
Development of an effective training machine using muscle activity information
}

\author{
Shunji Moromugi \\ Dept. of Mechanical Systems Engr. \\ Nagasaki University \\ 1-14 Bunkyo-machi, Nagasaki-shi \\ Nagasaki 852-8521, JAPAN \\ smoromug@nagasaki-u.ac.jp \\ Nobuou Matsuzaka \\ School of health science \\ Nagasaki University \\ 1-7-1 Sakamoto, Nagasaki-shi \\ Nagasaki 852-8520, JAPAN \\ nobuou@nagasaki-u.ac.jp
}

\author{
Seok-Hwan Kim \\ Rotem, LTD. \\ 462-18, Sam-Dong, Uiwang-Si \\ Gyunggi-Do, 437-718 \\ Korea \\ bigblaze@rotem.co.kr \\ Takakazu Ishimatsu \\ Dept. of Mechanical Systems Engr. \\ Nagasaki University \\ 1-14 Bunkyo-machi, Nagasaki-shi \\ Nagasaki 852-8521, JAPAN \\ ishi@nagasaki-u.ac.jp
}

\author{
Sung J. Yoon \\ Daihen Co. \\ 5-1 Minamisenrioka \\ Settsu-shi, Osaka 566-0021 \\ JAPAN \\ yoon@daihen.co.jp \\ Murray J. Lawn \\ Dept. of English and Computer Sci. \\ Nagasaki Junshin Catholic Univ. \\ 235 Mitsuyama, Nagasaki-shi \\ Nagasaki 852-8558, Japan \\ lawnmj@hotmail.com
}

\begin{abstract}
In the field of providing safe and effective equipment for physical training appropriate adjustment of loading levels for the targeted users is very important. Most training machines to date offer only fixed and coarse load adjustment. This paper details an advanced leg muscle training machine that has an intelligent load control system. The load control is provided by monitoring the user's muscular condition by means of a muscle stiffness sensor as well as pressure being exerted on the machine. The loading can then be adapted continuously to optimally suit the user's needs. The prototype demonstrated that the proposed machine has excellent load control abilities providing effective and safe training using dynamic load control and automatic restriction of loading levels based on the user's muscle contraction levels.
\end{abstract}

\section{INTRODUCTION}

Proper amounts of daily exercise helps people to maintain their good health. Machine trainings are recognized as one of the common ways to have exercise effectively as well as playing sports. It is reported that continuous physical training is quite effective even for elderly people to maintain good muscular tone as well as young people [1][2]. Therefore the machine training plays an important role for people of all ages in maintaining their health as well as for athletes to improve their performance.

However several problems with current training machines have been reported, two of the major problems are as follows. Firstly most training machines are designed primarily for able bodied persons, typically athletes. A system of wires and weights is typically used to provide a simple loading mechanism. Adjustment of the loading is however often quite difficult for people with less physical abilities such as elderly and disabled and the settings are often coarsely stepped. Therefore, such people often have troubles in adjustment of the loading levels. Secondly in regard to safety it is essential that people do not exceed their safe exercise limits. However even in the presence of licensed therapists or trainers this is difficult to judge. Clearly conventional training machines provide no inherent mechanism for judgment in this regard.
An intelligent training machine is prototyped with consideration for these problems. The prototyped machine presented in this paper provides knee extension training. This training machine has an electric motor based load control system. This system measures the user's muscle activity and pressure on the footplate during the exercise. The training load is controlled dynamically based on the user's muscular information to increase effectiveness and to ensure safety during the training.

\section{FEATURES OF THE PROPOSED TRAINING MACHINE}

The proposed training machine has the following advantages compared to conventional machines.

1. Ease of operation, the user or training assistant inputs the personal data necessary to define the range of the leg extension exercise and an estimation of the user's current physical strength.

2. Assurance of effective and safe training due to the use of a muscle stiffness sensor as well as exerted pressure sensor providing feedback to an intelligent load control system enhancing optimization of loading throughout the training period as the aspect of avoiding overloading, fatigue and injury is very important.

3. Data management. By maintaining records, user progress data can be made available for short term or long term analysis and for the subsequent planning of future training.

\section{SYSTEM CONFIGURATION}

The prototyped training machine is shown in Fig. 1. The mechanism consists of a sliding seat, an electronic motor, a stepping force sensor (SFS), a muscle stiffness sensor (MSS), a PC and a controller based on PIC (Peripheral Interface Controller) and FPGA(Flexibly Programmable Gate Allay). The user sits on the sliding seat and places their feet on the footplate. The seat is moved back and forward by the electric motor in such a way as to maintain a loading force defined by 


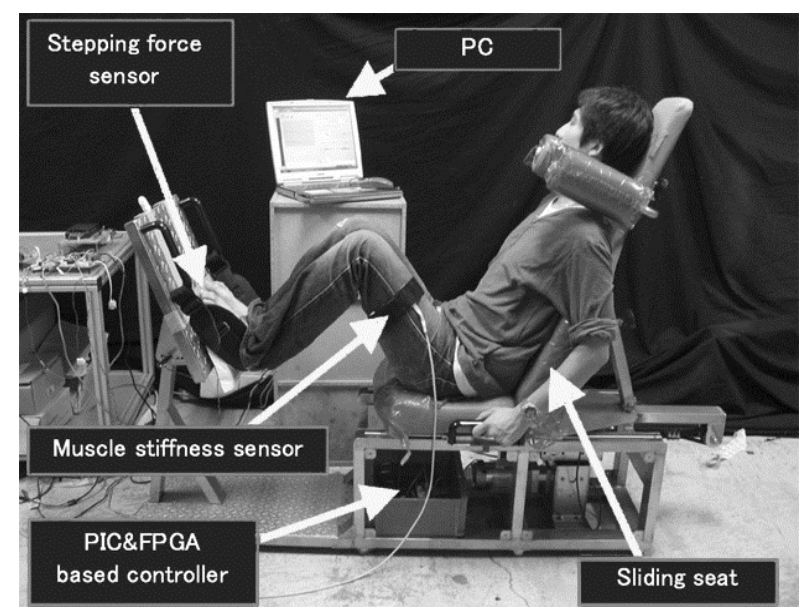

Fig. 1. Proposed leg press training machine

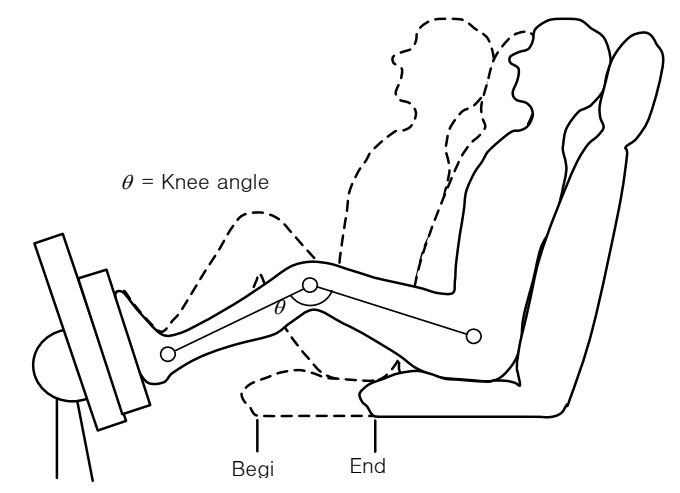

Fig. 2. Movement of the sliding seat during the exercise

the controller. Fig. 2 shows the movement of the sliding seat during the exercise. The user's force exerted on the footplate is measured by the SFS and the level of muscle activity is estimated by the MSS. The PC allows users to interact with the training machine. Users input their individual parameters before starting exercises or recall previously registered settings and are then provided with a graphical display of training data.

The mechanism automatically provides fine adjustment of load control in real time based on the user's exerted pressure on the footplate and information from the MSS. The system is currently programmed to provide training load during knee extension only that is no pressure is provided during the flexion. The seat automatically returns to the start position when the seat reaches to the end position after informing user the to relax their legs. The training machine can be stopped and restarted at any time, motor actuation is automatically stopped for safety if the user takes their feet off the footplate.

\section{MUSCLE STIFFNESS SENSOR}

In this study stress on the user's body is evaluated based on pressure data from the footplate and data from the MSS. The muscle stiffness sensor (MSS) shown in Fig. 3 was originally developed by the authors as a wearable man-machine interface to assist the control of human motion [3]. This sensor has two main components, a flat disk and a button in the center of the disk. The flat disk is $32 \mathrm{~mm}$ in diameter and $8 \mathrm{~mm}$ thick. The button is $6 \mathrm{~mm}$ in diameter and $4 \mathrm{~mm}$ in height. The weight of the sensor is $5 \mathrm{~g}$. The sensor is strapped to the user's thigh to monitor the level of muscle contraction of the muscle over which it is strapped. Reaction forces on the button and the sensor itself from the skin are measured by two pressure transducers, respectively. The level to which the target muscle is stiffened can be evaluated from the change of balance of the two forces measured. The contraction level of the muscle can be estimated from the sensor data because the muscle activity is directly related to the increased stiffness data. It is shown that there is a high correlation between the MSS data and Electromyogram (EMG) data.

Main advantages of the MSS compared with EMG based sensors are as follows:

1) Activity of a specific muscle is targeted.

2) Ease of installation. This sensor is located over the target muscle by simply wrapping an elastic belt or cuff over the sensor. The sensor can be worn even over clothes as shown in Fig. 1.

3) High robustness against mechanical and electrical interference. This sensor detects force on the sensor body and compensates for it automatically [3].

The muscle stiffness sensor has been used effectively as a man-machine interface for several types of wearable devices to assist human motion [4][5][6]. By using this sensor the training machine detects the user's level of muscular contraction with minimal interference to the user. The controller then adjusts the level of the training load in real time appropriately so as to provide both effective and safe training for the user via automatic load control.

\section{CONTROL SYSTEM}

The control system consists of a PC and a PIC/FPGA based controller. Fig. 4 shows the configuration of the control system. Before training begins users input their individual parameters into the $\mathrm{PC}$ so as to define the training machine start and end points (Fig. 2), also to define user ability or recall a previously input user profile. During training the PIC/FPGA based controller calculates the optimum motor torque based on leg angle, muscle activity from the MSS and footplate

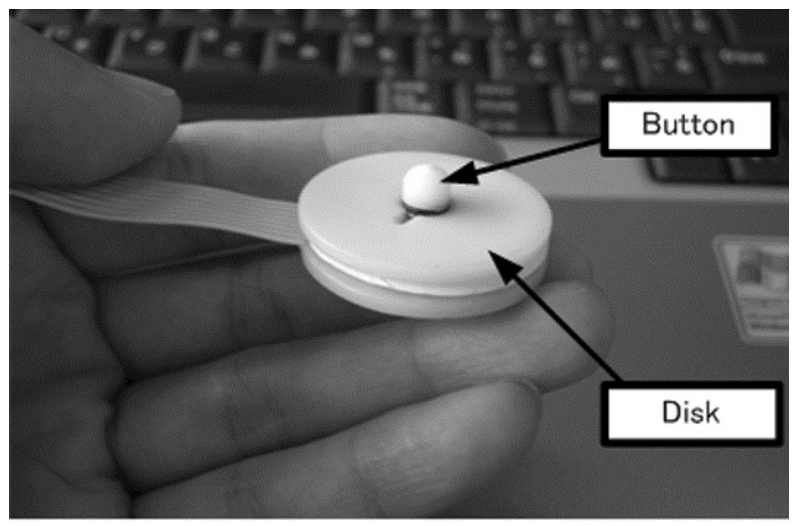

Fig. 3. Muscle stiffness sensor (MSS) 


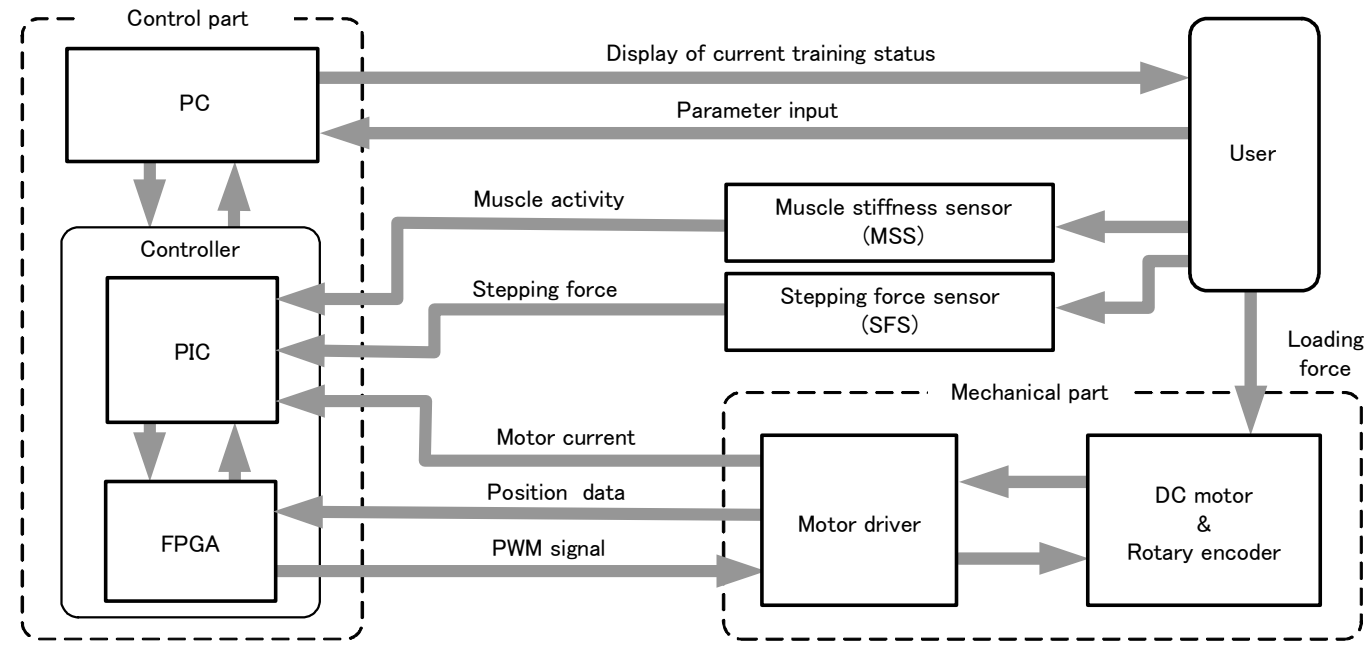

Fig. 4. Control system schematic

pressure. The controller then operates the electric motor via a PWM signal.

\section{EXPERIMENTS}

Several kinds of experiments have been carried out to evaluate the proposed system.

\section{A. Basic loading controllability}

In the first experiment the system's controllability of the loading level was evaluated through the loading control based on footplate pressure data. Examinees work on the knee extension exercise sitting on the proposed leg press training machine. The examinee is required to press the footplate three times. The target stepping force is set to $150[\mathrm{~N}], 300[\mathrm{~N}]$ and $450[\mathrm{~N}]$ respectively for each cycle. The machine monitors the electric current passing through the electric motor and estimates the amount of the load exerted on user's legs from the current data. The system controls the motor so that the estimated loading force (stepping force) maintains the desired level throughout the exercise. Proportional and Integral control algorithm is employed as a loading control strategy as shown in Fig.5.

From the experimental results in Fig. 6 it can be observed that the actual stepping force maintains levels close to $150[\mathrm{~N}]$, $300[\mathrm{~N}]$ and $450[\mathrm{~N}]$ respectively during each cycle by the loading control based on the feedback of the estimated stepping force. It is thus demonstrated that the proposed training machine has excellent controllability of loading force. The data from both the MSS and EMG sensor monitoring the quadriceps femoris muscle contraction also clearly showed increasing levels of the muscle stiffness contraction for increased loading.

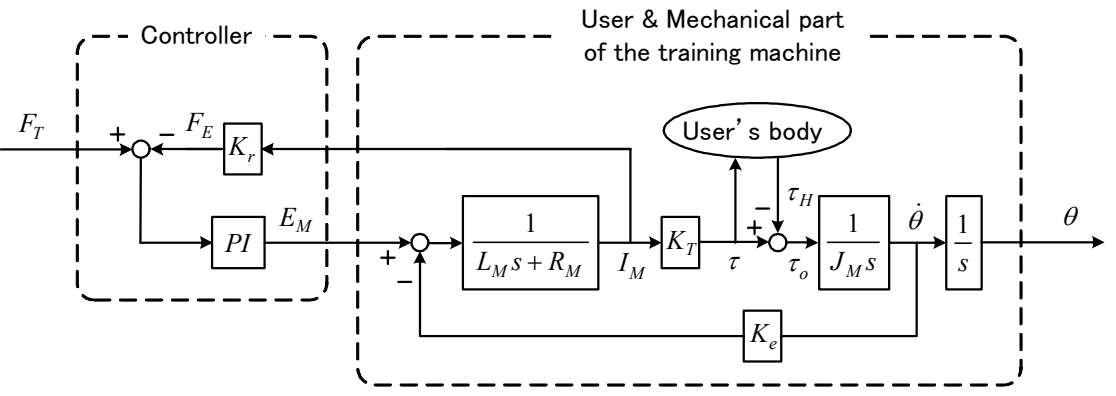

$F_{T}$ : Target stepping force

$F_{T}$ : Estimated stepping force

$E_{M}:$ Motor vol tage

$I_{M}:$ Motor current

$\tau:$ Output torque of motor

$\tau_{H}$ : Torque provided by users body to resist the seat movement

(Directly linked with stepping force) $\tau_{o}:$ Torque to move the sliding seat

$\theta$ : Motor rotation angle (Directly linked with knee angle)

$K_{r}$ : Experimental parameter to convert motor current to stepping force

$R_{M}$ : Armature resistance

$L_{M}:$ Armature inductance

$K_{T}$ : Torque constant

$J_{M}$ : Gross inertia moment

Fig. 5. Block diagram of the loading control 

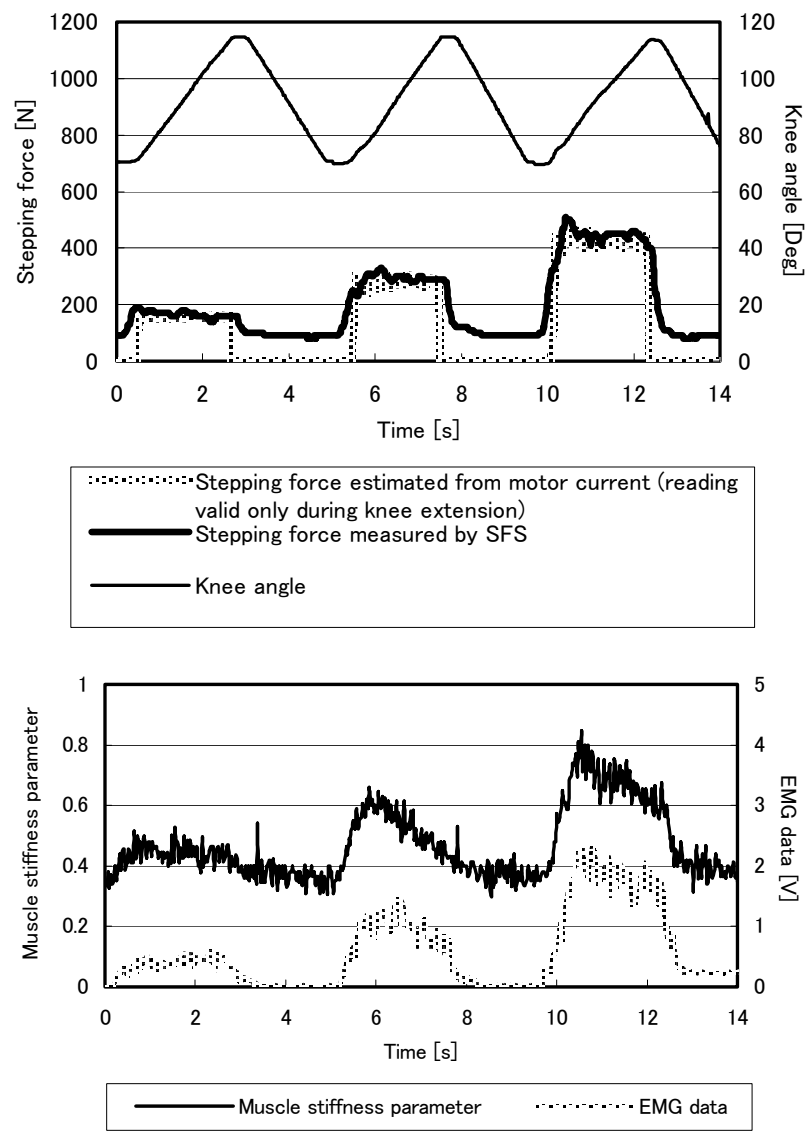

Fig. 6. Load control based on the monitored footplate pressure $(150 \mathrm{~N}, 300 \mathrm{~N}$ and $450 \mathrm{~N})$

\section{B. Examination of knee angle and exerted force}

In the second experiment the relationship between the knee angle, stepping force and the muscular contraction of the quadriceps femoris muscle predominantly used for knee extension is examined. The examinees were required to work on isometric knee flexion exercises. The footplate was fixed and examinees were requested to press the footplate with pre-specified stepping force. The stepping or exerted force and muscle activity for the knee flexion was recorded at various knee angles. The relationship between stepping force and muscle activity under various pre-specified knee angles is shown in Fig. 7. It is clear that the muscle stiffness data increases linearly under any knee angle condition as the stepping force is increased. It is also observed that muscle stiffness is higher at small knee angle region. This indicates that the user is required higher muscle contraction to exert the desired stepping force at lower knee angle (deeply flexed). These lines can be linearly approximated as shown in Fig.8.

\section{Load control based on muscle contraction}

In the third experiment the stepping force is controlled by the monitored stepping force and also the user's muscle
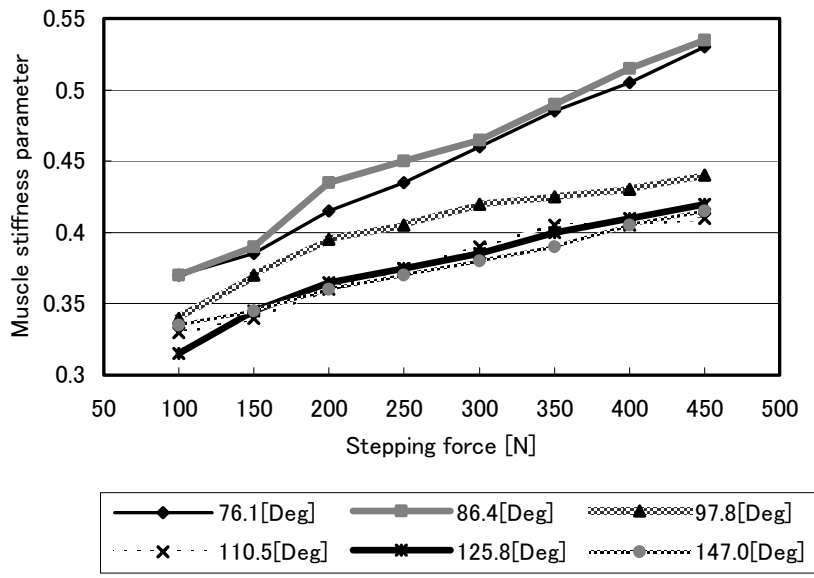

Fig. 7. Muscle stiffness vs. stepping force

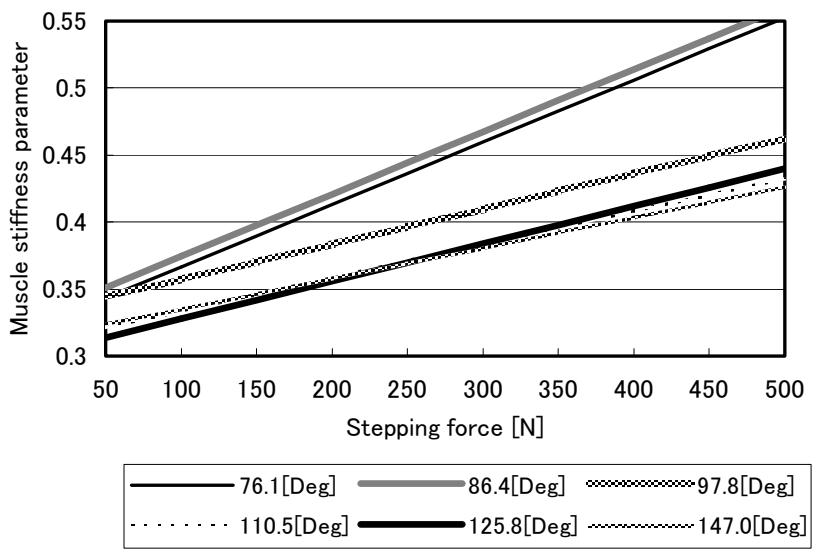

Fig. 8. Approximate expression of the experimental data

contraction level. The controller checks the level of the user's muscle contraction measured by the MSS and adjusts the target stepping force so that the level of muscle contraction doesn't exceed a pre-defined level for safety. In this experiment the stepping force was basically controlled so as to maintain a predefined level. Only when the muscle contraction exceeds the target level, the target stepping force is decreased so as to ensure the user exercises within predefined safe levels of muscle contractions.

A block diagram of the stepping force control is shown in Fig. 9, where $F_{d}$ is the desired stepping force and $F_{T}$ is the modified target stepping force. $F_{T}$ is determined as follows: $F_{T}$ equals $F_{d}$ while safety muscle contraction level is maintained. If for example the muscle contraction level exceeds the safe level $S_{T}$ and the current knee angle is 90 [Deg], a linear expression that describes the relationship between the stepping force and muscle contraction at the current knee angle $90[\mathrm{Deg}]$ is derived from the interpolating existing data (lines of knee angle 86.4[Deg] and 97.8[Deg]) shown in Fig. 8. Using this linear expression, it is clear that the safety muscle stiffness parameter $S_{T}$ can be achieved by setting the target stepping force $F_{T}$ to $F_{0}$ as shown in Fig.10. Therefore, $F_{T}$ can be described by 


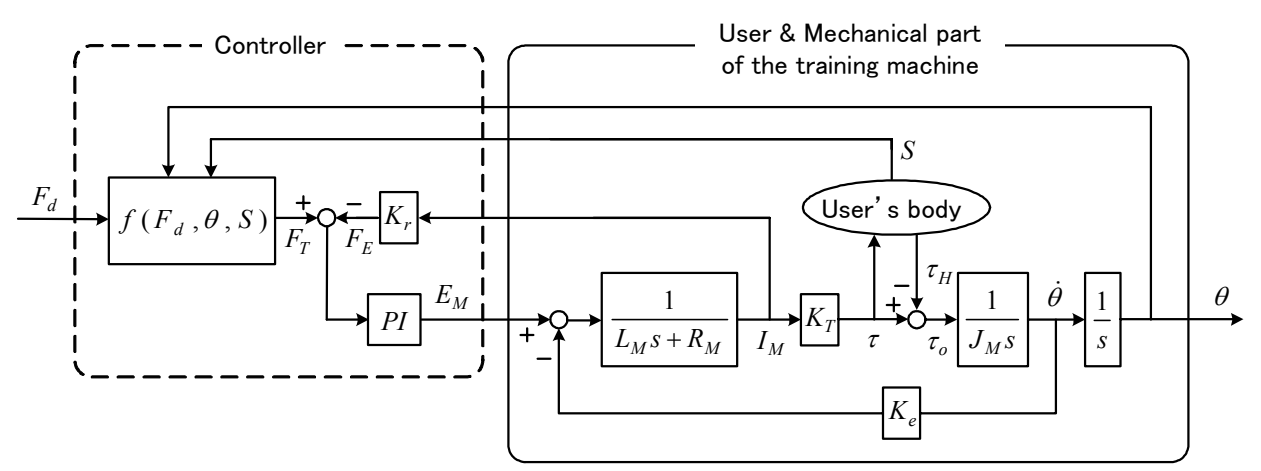

Fig. 9. Block diagram of load control with limitation of muscle contraction

$$
F_{T}=\left(\begin{array}{ll}
F_{0} & \left(S>S_{T}\right) \\
F_{d} & \left(S \leq S_{T}\right)
\end{array}\right.
$$

where $F_{0}$ is determined from the relationship shown in Fig.10.

In the experiment safe level of muscular contraction is increased in a stepwise fashion 28, 34, 40 and 46. Fig.11 shows the experimental data with the loading control set to limit the muscle contraction to specified levels. It is clear that

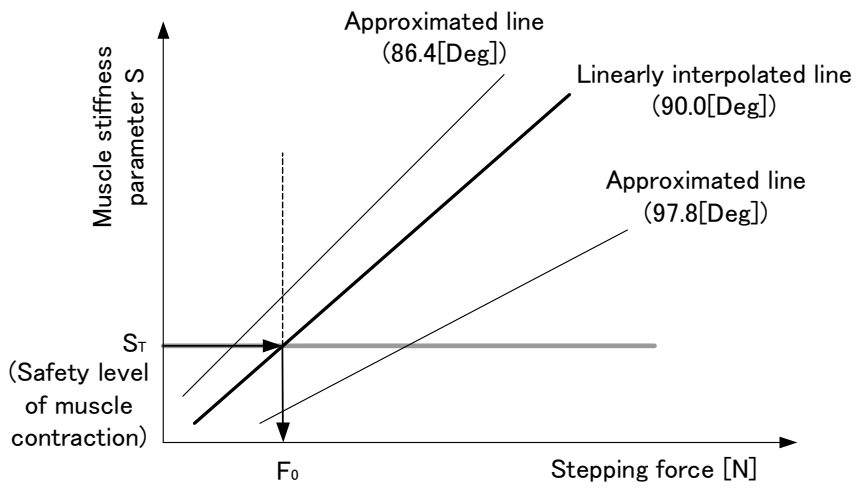

Fig. 10. Example of derivation of $F_{0}$ from the linearly interpolated line

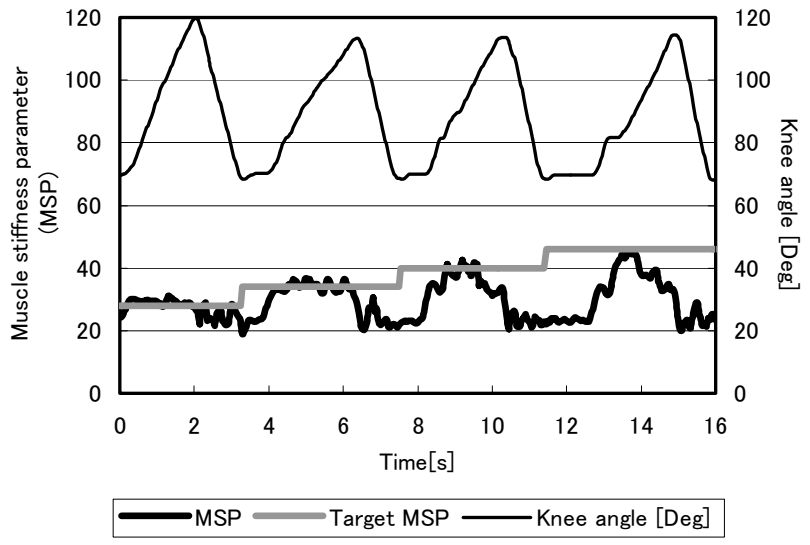

Fig. 11. Experimental data of load control based on muscle contraction the training load is controlled correctly and the load is maintained below the specified level of muscle contraction through out the exercise.

\section{CONCLUSION}

An advanced training machine is proposed. The training machine monitors exerted pressure on user's feet and the level of muscle contraction in order to enhance safety and effectiveness in training. Through several experiments it was demonstrated that the proposed machine allows training with loading control based on the condition of user's muscle. It was also demonstrated that the proposed training machine could automatically limit the user's knee extension exercise within a desired level of muscle contraction. This functionality would increase training effectiveness as well as training safety.

Further study is required with regard to loading pattern optimization for enhancing training effectiveness. Also the introduction of multiple sensors is currently being evaluated in order to increase the robustness and precision of the muscular stiffness monitoring system.

\section{ACKNOWLEDGMENT}

Authors would like to thank Mr. H. Hamanaka and Mr. Y. Matsumura of the NeeTec Hamanaka Co. and people of Daihen Co. for their great supports and contributions in this project.

\section{REFERENCES}

[1] K. Hakkinen, R. U. Newton, S. E. Gordon, M. McCormick, J. S. Volek, B. C. Nindl, L. A. Gotshalk, W. W. Campbell, W. J. Evans, A. Hakkinen, B. J. Humphries and W. J. Kraemer, "Changes in muscle morphology, electromyographic activity, and force production characteristics during progressive strength training in young and older men," Journals of Gerontology Series A: Biological Sciences and Medical Sciences, Vol. 53, Issue 6, 1998, B415-B423. 
[2] K. Hakkinen, W. J. Kraemer, R. U. Newton, M. Alen, "Changes in electromyographic activity, muscle fibre and force production characteristics during heavy resistance/power strength training in middle-aged and older men and women," Acta Physiol Scand., 171(1), 2001, pp.51-62.

[3] S. Moromugi, Y. Koujina, S. Ariki, A. Okamoto, T. Tanaka, M. Q. Feng and T. Ishimatsu, "Muscle stiffness sensor to control assisting device for disabled," Journal of Artificial Life and Robotics, Vol.8, No.1, 2004, pp.42-45.

[4] S. Moromugi, K. Izumi, T. Yoshimochi, T. Ishimatsu, T. Tanaka and M. Q. Feng, "Device for Assisting Grasping Function," in Proceedings of 17th International Conference on Control, Automation and Systems, Muju, Korea, Oct. 2002, pp.1250-1254.
[5] S. Moromugi, A. Okamoto, S. H. Kim, H. Tanaka, T. Ishimatsu and Y. Koujina, "Device for Assisting Grasping Function (2nd Report: Maneuverability Evaluation)," in Proceedings of 18th International Conference on Control, Automation and Systems, Gyeongju, Korea, Oct. 2003, pp.2665-2669.

[6] S. Moromugi, Y. Koujina, S. Ariki, T. Tanaka, Maria. Q. Feng and T. Ishimatsu, "Soft power suit for knee motion assistance," International Journal of Human-friendly Welfare Robotic Systems, Vol.4, No.4, 2003, pp.13-19. 